\title{
Rhodamine Labeled Albumin
}

National Cancer Institute

\section{Source}

National Cancer Institute. Rhodamine Labeled Albumin. NCI Thesaurus. Code C55827.

A tracer molecule applied in histo- and cytochemical as well as cytometry studies. Further, this conjug ate is used in quantitative and qualitative investigations into membrane permeability and molecular transfer mechanisms. 